\title{
Direct Observation of Aggregative Nanoparticle Growth: Kinetic Modeling of the Size Distribution and Growth Rate
}

Taylor J. Woehl ${ }^{1}$, Chiwoo Park ${ }^{2}$, James E. Evans ${ }^{3}$, Ilke Arslan ${ }^{3}$, William D. Ristenpart ${ }^{1}$, and Nigel D. Browning ${ }^{4}$

${ }^{1 .}$ Department of Chemical Engineering and Materials Science, University of California, Davis, Davis, CA 95616, USA

2.Department of Industrial and Manufacturing Engineering, Florida State University, Tallahassee, FL 32306

${ }^{3}$ Environmental and Molecular Sciences, Pacific Northwest National Laboratory, Richland, WA 99352, USA

4. Fundamental and Computational Sciences, Pacific Northwest National Laboratory, Richland, WA 99352, USA

Direct observations of solution-phase nanoparticle growth using in situ liquid transmission electron microscopy (TEM) have demonstrated the importance of mesoscale interactions, such as aggregation and coalescence, on the growth and final morphology of nanocrystals at the atomic and single nanoparticle scales $[1,2]$. To date, groups have quantitatively interpreted the mean growth rate of nanoparticles in terms of the Lifshitz-Slyozov-Wagner (LSW) model for Ostwald ripening [1, 3], but less attention has been paid to modeling the corresponding particle size distribution.

We used in situ liquid scanning TEM (STEM) to stimulate growth of hundreds of silver nanoparticles from a liquid precursor [4]. We measured the mean growth rate and particle size distribution of the nanoparticles during growth using custom image analysis algorithms [5]. The silver nanoparticles were observed to grow by monomer attachment, and remained mobile on the surface of the fluid cell window often aggregating with neighboring particles. The total number of nanoparticles after nucleation occurred decreased by $\sim 30 \%$ over a few minutes due to aggregation.

Here we demonstrate that silver nanoparticles grow by a length-scale dependent mechanism, where single particles grow by monomer attachment but ensemble-scale growth is dominated by aggregation. Although the observed mean nanoparticle growth rate is consistent with the LSW model, we show that the corresponding particle size distribution is broader and more symmetric than predicted by LSW. Following direct observations of aggregation, we interpret the ensemble-scale growth using Smoluchowski kinetics and demonstrate that the Smoluchowski model quantitatively captures the mean growth rate and particle size distribution. Likewise, our observations of length-scale dependent nanoparticle growth emphasize the need to quantify and correlate the contributions of growth mechanisms at different length scales to help tune the overall properties of functional nanostructures, biomineralized nanocrystals, heterogeneous catalysts, and other complex nanoscale ensembles formed by mesoscale interactions [6].

\section{References:}

[1] H. M. Zheng, et al, Science 324 (2009), p. 1309

[2] J. M. Yuk, et al, Science 336 (2012), p. 61. 
[3] Y. Liu, et al, Chemistry of Materials 25 (2013), p. 2927.

[4] T. J. Woehl, et al, Nano Letters 14 (2013), p. 373.

[5] C. Park, et al, IEEE Transactions of Pattern Analysis and Machine Intelligence 35 (2013), p. 669. [6] J.E.E. and N.D.B. acknowledge NIH funding support from grant number 5RC1GM091755. N.D.B. acknowledges DOE funding support from grant number DE-FG02-03ER46057. I.A. acknowledges support from the Presidential Early Career Award for Scientists and Engineers. C.P. acknowledges support from the FSU COFRS Award 032968, the Ralph E. Powe Junior Faculty Enhancement Award, and NSF-CMMI-1334012. A portion of this work is part of the Chemical Imaging Initiative at Pacific Northwest National Laboratory under Contract DE-AC05-76RL01830 operated for DOE by Battelle.
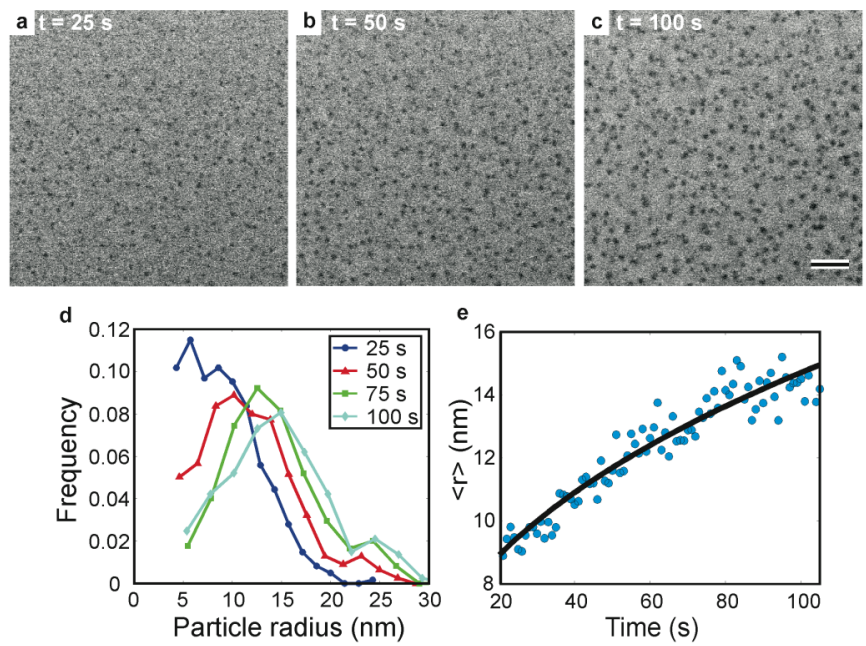

Figure 1. (a)-(c) Time lapsed series of BF-STEM images showing growth of an ensemble of silver nanoparticles starting at 25 seconds from the initial irradiation ( $20 \mathrm{pA}$ beam current). The scale bar in (c) is $200 \mathrm{~nm}$. (d) Particle size distribution (PSD) of the ensemble for various times; the PSD's are normalized by their total integral to yield a probability density function. (e) Mean nanoparticle radius as a function of time. The black line is the result of a power law fit of the form $\langle\mathrm{r}\rangle=\mathrm{Kt}^{\langle\beta\rangle}$, where linear regression yielded $\langle\beta\rangle=0.31 \pm 0.01$.

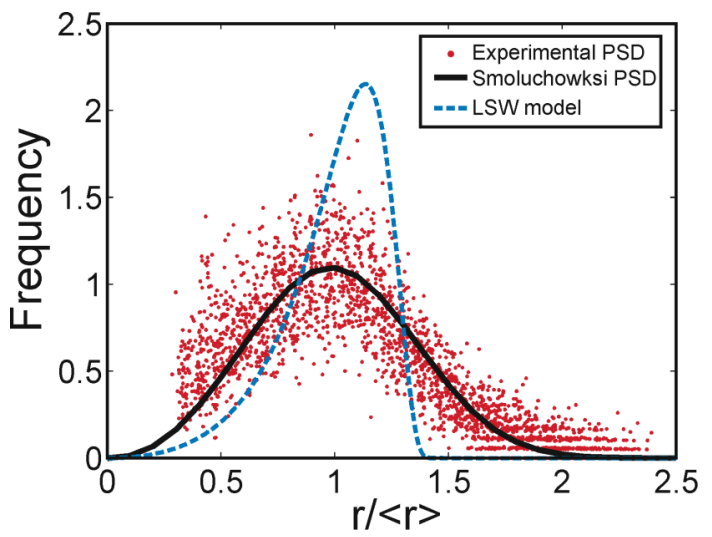

Figure 2. The scaled nanoparticle PSD measured for all times from $t=15-105 \mathrm{~s}$. The dashed blue curve is the LSW PSD (sum of squared errors SSE = 1550), and the solid black curve is the Smoluchowski PSD with $\left\langle\beta_{\mathrm{PSD}}>=<\beta>=0.31\right.$ (SSE $\left.=78\right)$. Nanoparticle radii are normalized to the respective mean nanoparticle radius for that time. 